\title{
PROMOVENDO O USO DO AMBIENTE VIRTUAL DE APRENDIZAGEM ESTUDO SOBRE A NÃO ADESÃO DO USO DO AMBIENTE VIRTUAL DE APRENDIZAGE E A SUA SUBUTILIZAÇÃO POR UMA PARCELA DOS DOCENTES DA FUNDAÇÃO GETULIO VARGAS
}

\author{
SÃO PAULO/SP MARÇO/2018
}

\author{
Flavio Roberto Bueno de Camargo－FGV - flavioroberto15@gmail.com \\ Tipo: Investigação Científica (IC) \\ Natureza: Relatório Final de Pesquisa \\ Categoria: Pesquisa e Avaliação \\ Setor Educacional: EDUCAÇÃO SUPERIOR
}

\begin{abstract}
RESUMO
O uso de Ambientes Virtuais de Aprendizagem (AVA) em instituições do ensino superior visa modernizar e agregar valor ao processo de ensino e aprendizagem. Desta forma, após 14 anos do uso de diversos AVA's, a Fundação Getulio Vargas (FGV) decidiu implantar um AVA único para suas oito escolas. O desafio da implantação foi tratado com grande planejamento e contou com os diferenciais do novo ambiente, bem como uma estratégia simples, mas de intensa comunicação e capacitação aos docentes. Apesar do esforço realizado, há uma parcela dos docentes que não utilizam a nova plataforma ou há utilizam de forma superficial. O presente trabalho tem como objetivo analisar o índice de utilização desta plataforma. Adicionalmente, pretende-se identificar os pontos críticos da falta de adesão por parte dos docentes. Com o resultado deste trabalho foram revistas as premissas que levam à compreensão da adequação do sistema e, também a elaboração de um plano de ação que contribua para o crescimento da adesão à nova ferramenta.
\end{abstract}

Palavras-chave: Ambiente Virtual de Aprendizagem; Comunicação e Capacitação; Implantação de Tecnologia Educacional; Adesão.

\section{AGRADECIMENTOS}

AGRADEÇO A TODOS QUE POSSIBILITARAM O DESENVOLVIMENTO DESSA PESQUISA. EM ESPECIAL AO RICARDO GUTH, PAULO GOES, DIANA ABREU, MINHA ORIENTADORA MARTHA SAVASTANO E O APOIO DE MINHA ESPOSA E FAMILIARES. 


\section{Introdução}

O uso de tecnologias educacionais, especificamente em Ambientes Virtuais de Aprendizagem (AVA) na Fundação Getulio Vargas (FGV) não é algo novo. A FGV, por meio de suas escolas e do Programa de Educação Executiva FGV online, está no mercado trazendo inovação há mais de 20 anos, não somente oferecendo cursos e disciplinas totalmente online, mas principalmente com 0 uso das tecnologias educacionais para o apoio ao ensino e aprendizagem presencial.

A FGV agrega oito escolas com características distintas em duas grandes cidades, Rio de Janeiro e São Paulo, e até dezembro de 2014 mantinha quatro Ambientes Virtuais de Aprendizagem (AVA), sendo que cinco escolas compartilhavam o mesmo AVA e outras três com pouca experiência e/ou experiências mal sucedidas no uso do AVA.

No final de 2014, após uma vasta pesquisa de mercado, foi adquirido um novo AVA. Foi implantado o novo AVA, cujo nome original é Brightspace, mas optou-se por intitulá-lo como novo ECLASS para não vincular com o nome comercial. Este novo nome faz alusão a um dos AVA antigo e que era mais difundido na FGV. Como resultado no início de 2015 o novo ECLASS foi implementado nas oito escolas da FGV, sendo desligados oficialmente os quatro sistemas anteriores. Importante ressaltar que pelo menos dois deles estavam consolidados há 12 anos.

\section{Apresentação do problema, do objetivo e sua relevância}

O problema a ser solucionado e que orienta este estudo de caso foi assim descrito " $\mathrm{A}$ não adesão do uso do AVA e a sua subutilização por uma parcela dos docentes da Fundação Getulio Vargas". Diante deste problema, este estudo buscou identificar suas possíveis causas e apresentar possíveis sugestões de soluções.

O presente trabalho tem como objetivo analisar o índice de utilização da plataforma ECLASS pelos docentes da FGV. Adicionalmente, pretende-se identificar os pontos críticos da falta de adesão por parte dos docentes. Com o resultado deste trabalho foram revistas as premissas que levam à compreensão da adequação do sistema e, também a elaboração de um plano de ação que contribua para o crescimento da adesão à nova ferramenta. Diante da situação identificada na tabela 1 , as quais indicam um alto índice do uso do ECLASS após sua implementação, também é possível perceber que ainda há uma parcela dos docentes da FGV que não utilizam.

Tabela 1 - Dados de utilização do novo ECLASS 


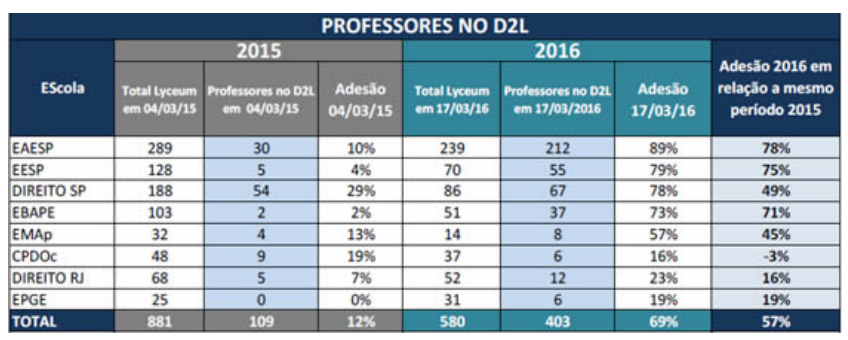

Fonte: elaboração do autor

Diante destes dados nota-se a necessidade de ações para conseguir aumentar a adesão ao uso do ECLASS, principalmente nas unidades com índice abaixo de 50\%. A FGV almeja conquistar esta parcela de docentes que ainda não utilizam o ECLASS, e este estudo contribuirá com as medidas necessárias para que este objetivo seja alcançado.

\section{Hipóteses}

De acordo com Luna (1997), com base em análises do conhecimento disponível, o pesquisar acaba "apostando" naquilo que pode surgir como resultado de sua pesquisa. Uma vez formulado o problema, é proposto uma resposta suposta, provável e provisória, que seria o que ele acredita ser plausível como solução do problema. Dito isso, abaixo há respostas supostas para o problema já relatado:

1. Docentes com maior idade podem usar menos o ECLASS, 2. Docentes com mais tempo de casa podem usar menos o ECLASS, 3. Docentes de uma determinada escola podem usar menos o ECLASS, 4. A falta de adesão e subutilização pode ter ocorrido pela falta de comunicação e divulgação da plataforma e seus benefícios;

5. A percepção de benefícios está diretamente relacionada à ciência dos professores quanto às funcionalidades existentes.

\section{Organização do trabalho}

Este trabalho conta com análises dos métodos de correlação de informações que é um dos métodos utilizados para desenvolver a pesquisa que norteia este projeto. Este método irá comprovar o problema identificado que é "a não adesão do uso do AVA e a sua subutilização por uma parcela dos docentes da Fundação Getulio Vargas", bem como, fornecer ferramentas para aplicação de um plano de ação/decisão que será abordado ao final deste estudo.

"A ciência estatística fornece ferramentas muito utilizadas para inferir resultados a partir de uma base de dados, que podem conter informações qualitativas ou quantitativas, com meios diferentes de coletar informações e demonstrar os dados" (MARTINS; DOMINGUES, 2014). 
Desse modo, a condução da pesquisa está embasada nos conceitos e nos desafios metodológicos que incluem a tomada de decisão sobre a forma do fluxograma a seguir:

1. Determinar as necessidades / problemas; 2. Elaborar e avaliar o questionário; 3. Determinar amostragem; 4. Aplicar questionário; 5. Analisar dados e 6. Conclusão/ Plano de melhoria

Seguindo os critérios conceituais da tomada de decisão do pesquisador, o trabalho foi planejado da seguinte forma:

1. Foram identificados quais seriam os pontos chaves que justificariam a não adesão do ECLASS por uma parcela dos docentes da FGV; 2. Foram levantados os recursos existentes do novo ECLASS, o que permitiria medir a subutilização do ambiente virtual de aprendizagem; 3. Foi estruturado o questionário estabelecendo as perguntas e alternativas de resposta, de forma que se consiga realizar posteriormente uma análise estatística para resolver o problema; 4. Foi criado o questionário na ferramenta Formulários Google e 5. Foi divulgada e aplicada a pesquisa a todos os docentes que compõe as oito escolas da FGV. A divulgação foi consolidada através de e-mail institucional pela área responsável da administração do ECLASS que é a Coordenação de Tecnologias Educacionais (CTE), e dessa forma fortalecendo o incentivo para que todos os docentes respondessem ao questionário, assim contribuindo para melhorias no novo sistema.

\section{A Pesquisa}

Para identificar o nível de utilização da plataforma ECLASS e os pontos críticos que levam a não adesão por uma parcela dos professores, foi realizada uma pesquisa com base no questionário anexo a este documento e encaminhada para a população total de professores da FGV, em seus vários níveis educacionais (graduação, pós-graduação, mestrado, doutorado e cursos de extensão) e das escolas (CPDOC, Direito RJ, Direito SP, EAESP, EBAPE, EESP, EMAP e EPGE). A pesquisa foi encaminhada para 835 professores, dos quais $150,18,0 \%$ do total, responderam efetivamente todas as perguntas formuladas.

\section{Análise estatística}

A partir da amostra disponível, é possível verificar que $27 \%$ dos professores pesquisados pouco utilizam a plataforma. Ou seja, 40 dos 148 docentes que responderam à pesquisa afirmaram que não utilizam o ECLASS ou que o utilizam menos de uma vez por semana.Através desses números foram verificadas as hipóteses para entender o que leva essa parcela do corpo docente a não utilizar a plataforma. 
Confrontando a informação de frequência de uso do ECLASS com os demais dados informados pelos professores, foi feito uma busca para encontrar respostas para essa indagação.

\section{Docentes com maior idade podem usar menos o ECLASS}

A primeira hipótese a ser testada indaga se a idade informada pelos professores se relaciona diretamente com a frequência de utilização do ECLASS. Essa hipótese pressupõe que os professores com idade mais avançada poderiam preferir métodos tradicionais a ferramentas atuais para lecionar. Para obter uma resposta a essa pergunta, foi relacionada à variável de idade, definida em faixa etária com intervalo de dez anos entre cada categoria, e a variável de frequência de utilização do ECLASS, que pede ao pesquisado para escolher entre as seguintes opções qual a melhor se adequa ao seu perfil de uso: não utilizo, utilizo pelo menos uma vez ao mês, pelo menos uma vez a cada 15 dias, uma vez por semana ou duas vezes por semana.

Contudo, o resultado obtido indica não existir relação entre essas duas variáveis. Com efeito, a média de idade calculada para os professores que pouco utilizam a plataforma não difere estatisticamente da média dos professores que afirmaram utilizar o ECLASS pelo menos uma vez por semana.

Tabela 2 - Relação entre Faixa Etária e Frequência de Utilização do ECLASS.

\begin{tabular}{l|c|c|c|c}
\hline \multirow{2}{*}{ Faixa Etária } & \multicolumn{2}{|c|}{ Não utilizam ou utilizam pouco } & \multicolumn{2}{c}{ Utilizam com frequência } \\
\cline { 2 - 5 } & Frequência (ni) & $\begin{array}{c}\text { Frequência Relativa } \\
\text { (fi) }\end{array}$ & Frequência (ni) & $\begin{array}{c}\text { Frequência Relativa } \\
\text { (fi) }\end{array}$ \\
\hline Até 30 anos & 2 & $5,0 \%$ & 3 & $2,8 \%$ \\
De 31 a 40 anos & 11 & $27,5 \%$ & 24 & $22,2 \%$ \\
De 41 a 50 anos & 7 & $17,5 \%$ & 29 & $26,9 \%$ \\
De 51 a 60 anos & 11 & $27,5 \%$ & 32 & $29,6 \%$ \\
Acima de 60 anos & 9 & $22,5 \%$ & 20 & $18,5 \%$ \\
\hline Total Geral & $\mathbf{4 0}$ & $\mathbf{1 0 0} \%$ & $\mathbf{1 0 8}$ & $\mathbf{1 0 0} \%$ \\
\hline Fonte: Questionánio enviado aos professores da FGV. Elaboração própria.
\end{tabular}

\section{Docentes com mais tempo de casa podem usar menos o ECLASS}

Após a rejeição da primeira hipótese, será avaliado se a frequência de utilização do ECLASS está relacionada não à idade do professor, mas sim ao tempo de casa. Desta vez, a hipótese pressupõe que os professores que trabalham há mais tempo na FGV teriam maior resistência às seguidas mudanças de plataformas de apoio às aulas presenciais e por isso deixariam de usar a ferramenta mais recente. Antes, porém, foi considerado ser de grande relevância testar como o tempo de casa informado pelos professores pesquisados se relaciona com a idade. Outra hipótese trabalhada é de que o tempo de casa é diretamente proporcional à idade de cada professor e, assim sendo, o resultado da questão anterior tenderia a ser pouco diferente do observado para a relação entre faixa etária e utilização do ECLASS. 
Com efeito, a combinação entre o tempo de casa dos professores e os seus respectivos anos de vida mostra uma relação direta positiva entre essas duas variáveis. $O$ índice de correlação entre essas duas informações é próximo a 68\%.

Gráfico 1 - Relação entre Faixa Etária e Tempo de Casa (índices padronizados).

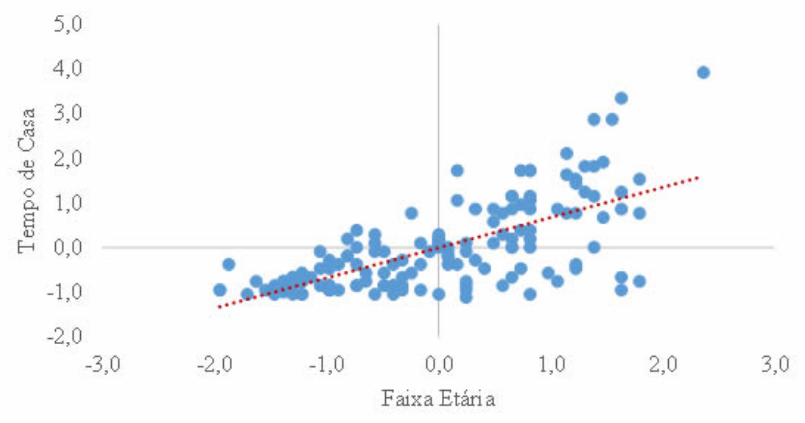

Assim sendo, é de se esperar que a segunda hipótese trabalhada, a que relaciona a não utilização do ECLASS com o tempo de casa dos professores, também seja rejeitada. Para medirmos a relação entre a frequência de utilização do ECLASS e o tempo de casa, distribuímos esta informação em faixas com o intervalo de cinco anos entre cada categoria.

Tabela 3 - Relação entre Tempo de Casa e Frequência de Utilização do ECLASS

\begin{tabular}{|c|c|c|c|c|}
\hline \multirow[b]{2}{*}{ Tempo de Casa (em anos) } & \multicolumn{2}{|c|}{ Não utilizam ou utilizam pouco } & \multicolumn{2}{|c|}{ Utilizam com frequência } \\
\hline & Frequência (ni) & $\begin{array}{l}\text { Frequência } \\
\text { Relativa (i) }\end{array}$ & Frequência (ni) & $\begin{array}{l}\text { Frequência Relativa } \\
\text { (fi) }\end{array}$ \\
\hline Até 5 anos & 20 & $50,0 \%$ & 34 & $31,5 \%$ \\
\hline De 6 a 10 anos & 5 & $12,5 \%$ & 21 & $19,4 \%$ \\
\hline De 11 a 15 anos & 7 & $17,5 \%$ & 20 & $18,5 \%$ \\
\hline De 16 a 20 anos & 2 & $5, \% \%$ & 8 & $7,4 \%$ \\
\hline De 21 a 25 anos & 1 & $2,5 \%$ & 12 & $11,1 \%$ \\
\hline Acima de 25 anos & 5 & $12,5 \%$ & 13 & $12,0 \%$ \\
\hline Total Geral & 40 & $100 \%$ & 108 & $100 \%$ \\
\hline
\end{tabular}

\section{A falta de adesão e subutilização pode ter ocorrido pela falta de comunicação e divulgação da plataforma e seus benefícios}

Com efeito, diferentemente do ocorrido com as duas hipóteses anteriores, é possível observar pelas informações trabalhadas a partir do cruzamento entre o benefício percebido pelo professor na utilização do ECLASS e a frequência de uso dessa ferramenta, que essas duas variáveis têm relação direta entre si. O professor que não percebe os benefícios do ECLASS tende a não utilizar a ferramenta. Ou, quanto menor a percepção do professor quanto aos benefícios da ferramenta, menos ele a utiliza.

Para identificar a percepção de cada professor quanto aos benefícios da plataforma, o questionário solicita ao pesquisado para enumerar numa escala de 1 (discordo plenamente) a 5 (concordo plenamente) o nível de concordância com a seguinte 
afirmação: "Em sua opinião, o sistema ECLASS propicia benefícios no apoio à sua aula presencial?".

Tabela 4 - Distribuição conjunta da Percepção de benefícios do ECLASS e Frequência de utilização

\begin{tabular}{|c|c|c|c|c|c|c|}
\hline \multirow{2}{*}{$\begin{array}{l}\text { OE-Class traz benefic bs a } \\
\text { aula presencial? }\end{array}$} & \multicolumn{5}{|c|}{ 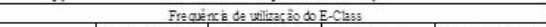 } & \multirow[b]{2}{*}{ Total Gem } \\
\hline & Nàoutilio & 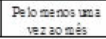 & \begin{tabular}{|l|} 
Palo menos vasa \\
vaza cata 15 das
\end{tabular} & 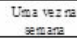 & 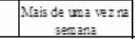 & \\
\hline Discodoplanaments & $1,4 \%$ & $0,0 \%$ & $0,7 \%$ & $0,0 \%$ & $1,4 \%$ & $3,4 \%$ \\
\hline Discordo & $0,0 \%$ & $2,0 \%$ & $0,0 \%$ & $1,4 \%$ & $0,7 \%$ & $4,1 \%$ \\
\hline Nen disx orda nen concordo & $2,7 \%$ & $1,4 \%$ & $2,7 \%$ & $4,1 \%$ & $2,7 \%$ & $13,5 \%$ \\
\hline Concordo & $0,7 \%$ & $5,4 \%$ & $2,7 \%$ & $6,1 \%$ & $21,6 \%$ & $36,5 \%$ \\
\hline Concordo planaments & $0,7 \%$ & $1,4 \%$ & $5,4 \%$ & $8,8 \%$ & $26,4 \%$ & $42,6 \%$ \\
\hline Iotal Geral & $5,4 \%$ & $10,1 \%$ & $11,5 \%$ & $20,3 \%$ & $52,7 \%$ & $100,0 \%$ \\
\hline & & & & & Inalos de correlaça: & 34,80996 \\
\hline
\end{tabular}

A percepção de benefícios está diretamente relacionada à ciência dos professores quanto às funcionalidades existentes

Se a não percepção dos benefícios que o ECLASS proporciona à aula presencial é um dos fatores que ajudam a explicar a não adesão à plataforma por parte dos professores, o que então levaria esses docentes a não identificar os pontos positivos da plataforma? Com as informações obtidas na pesquisa e a partir da amostra de dados disposta, foi encontrada uma relação forte entre a opinião dos professores sobre o ECLASS e a ciência quanto às funcionalidades disponíveis na plataforma.

Gráfico 2 - Quantidade de funcionalidades conhecidas pelos professores por grupo de concordância em relação ao benefício do ECLASS no apoio à aula presencial

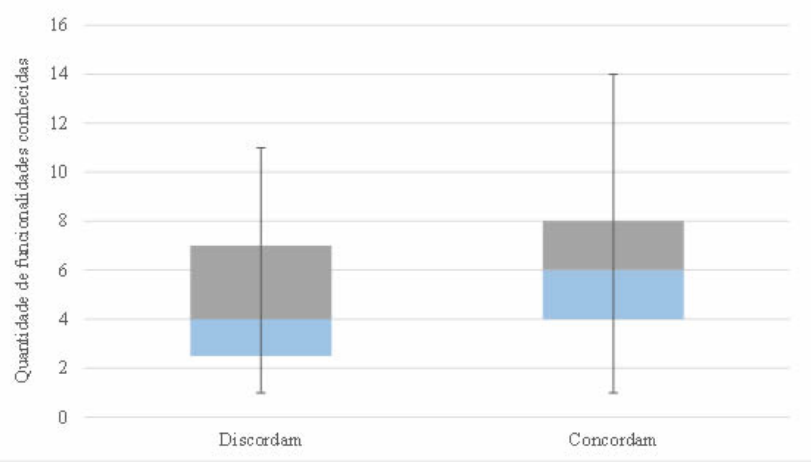

Quanto menor é o conhecimento das funcionalidades do ECLASS, maior é a discordância com a afirmação de que o ECLASS traz benefícios à aula presencial.

Tabela 5 - Distribuição conjunta da Percepção de benefícios do ECLASS e Quantidade de funcionalidades conhecidas. 


\begin{tabular}{|c|c|c|c|c|c|c|}
\hline \multirow{2}{*}{$\begin{array}{l}\text { Quantita de de fincibalitades } \\
\text { qe oprdessur tan ciancia }\end{array}$} & \multicolumn{5}{|c|}{ 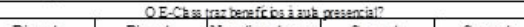 } & \multirow[b]{2}{*}{ Total Geral } \\
\hline & $\begin{array}{l}\text { Discordo } \\
\text { dlenazanta }\end{array}$ & $\begin{array}{l}\text { Discordo } \\
\text { arciatenats. }\end{array}$ & $\left|\begin{array}{c}\text { Nes discordo, nen } \\
\operatorname{cosecodo}\end{array}\right|$ & $\begin{array}{l}\text { Concondo } \\
\text { carciabeante }\end{array}$ & $\begin{array}{l}\text { Concordo } \\
\text { ckanentas }\end{array}$ & \\
\hline 1 & $1,4 \%$ & $0,7 \%$ & $2,0 \%$ & $1,4 \%$ & $0,7 \%$ & $6,1 \%$ \\
\hline 2 & $0,0 \%$ & $0,7 \%$ & $0,7 \%$ & $1,4 \%$ & $0,7 \%$ & $3,4 \%$ \\
\hline 3 & $1,4 \%$ & $0,0 \%$ & $3,4 \%$ & $0,7 \%$ & $6,8 \%$ & $12,2 \%$ \\
\hline 4 & $0,7 \%$ & $0,0 \%$ & $1,4 \%$ & $8,1 \%$ & $4,7 \%$ & $14,9 \%$ \\
\hline 5 & $0,0 \%$ & $0,7 \%$ & $0,0 \%$ & $6,1 \%$ & $4,7 \%$ & $11,5 \%$ \\
\hline 6 & $0,0 \%$ & $0,0 \%$ & $2,0 \%$ & $6,1 \%$ & $6,1 \%$ & $14,2 \%$ \\
\hline 7 & $0,0 \%$ & $0,7 \%$ & $0,7 \%$ & $1,4 \%$ & $1,4 \%$ & $4,1 \%$ \\
\hline 8 & $0,0 \%$ & $0,7 \%$ & $1,4 \%$ & $2,7 \%$ & $6,8 \%$ & $11,5 \%$ \\
\hline 9 & $0,0 \%$ & $0,7 \%$ & $0,0 \%$ & $2,0 \%$ & $4,1 \%$ & $6,8 \%$ \\
\hline 10 & $0,0 \%$ & $0,0 \%$ & $1,4 \%$ & $1,4 \%$ & $2,0 \%$ & $4,7 \%$ \\
\hline 11 & $0,0 \%$ & $0, \infty \%$ & $0,7 \%$ & $0,7 \%$ & $2, \infty \%$ & $3,4 \%$ \\
\hline 12 & $0,0 \%$ & $0, \infty \%$ & $0,0 \%$ & $1,4 \%$ & $2, \infty \%$ & $3,4 \%$ \\
\hline 13 & $0,0 \%$ & $0, \infty \%$ & $0,0 \%$ & $2,0 \%$ & $0,7 \%$ & $2,7 \%$ \\
\hline 14 & $0,0 \%$ & $0,0 \%$ & $0,0 \%$ & $1,4 \%$ & $0,0 \%$ & $1,4 \%$ \\
\hline Iotal Ceral & $3,4 \%$ & $4,1 \%$ & $13,5 \%$ & $36,5 \%$ & $42,6 \%$ & $100,0 \%$ \\
\hline
\end{tabular}

Essa informação corrobora a hipótese de que a comunicação pode ter sido falha, não atingindo o corpo docente da FGV em sua plenitude.

\section{Docentes de uma determinada escola podem usar menos o ECLASS}

Também foi verificado que a distribuição entre escolas dos professores que pouco utilizam o ECLASS é mais heterogênea que a própria distribuição entre o total dos 148 professores, o que nos permite inferir que essa possível falha de comunicação não foi um caso isolado em uma determinada escola da FGV.

\section{Proposta}

Como proposta serão realizadas ações, que serão devidamente descritas neste capítulo, com o objetivo de reduzir a não adesão dos docentes da FGV de $31 \%$ dos docentes para 11\%. Esta redução tem como meta ocorrer em 1 ano tendo início em julho de 2018 e seu encerramento deve ocorrer em julho de 2019. Após o encerramento desta ação será realizada uma nova pesquisa a fim de medir novamente a taxa de adesão dos docentes da FGV pelo novo AVA, e então verificar se houve sucesso nas ações tomadas ao longo do ano obtendo uma redução da não adesão.

\section{Pesquisa Exploratória}

Para escolher quais ações devem ser realizadas a fim de diminuir a taxa de não adesão para $11 \%$ e ter maior eficácia, assertividade e eficiência, foi realizado uma pesquisa exploratória com uma amostragem dos docentes que compõem o universo dos que não aderiram o novo AVA. Vale ressaltar que as pesquisas exploratórias, segundo Gil (1999) visam proporcionar uma visão geral de um determinado fato, do tipo aproximativo.

Foi usado uma amostragem de $10 \%$ dos docentes que faziam parte dos $27 \%$ pesquisados e que pouco utilizam a plataforma. Ou seja, foram selecionados 4 docentes dos 40 que responderam à pesquisa e afirmaram que não utilizam o ECLASS. Para proporcionar uma visão geral do fato, do tipo aproximativo a pesquisa realizada pessoalmente consistia em responder as seguintes questões: 
1. Como o docente leciona sua aula, ou seja, qual é a dinâmica utilizada? 2. Quais suas necessidades de interação com os alunos? Por exemplo, há a necessidade de comunicação extra aula presencial, de compartilhar material, ou de realizar atividades? 3. O docente já faz uso de algum recurso tecnológico para em sua aula? Se sim quais? Se não, qual motivo? 4. O docente conhece o ECLASS? Se sim, já usou? Se não usou, qual o motivo por não usar?

\section{Ações da proposta}

Analisando as respostas da pesquisa exploratória foi possível concluir que os quatros professores entrevistados não faziam o uso do ECLASS por não ter conhecimento dos benefícios que ele poderia propiciar para sua aula. Essa informação, mais uma vez, corrobora a hipótese de que a comunicação tem sido falha, não atingindo o corpo docente da FGV em sua plenitude. Após esta conclusão é necessário tomar ações que possam mudar este quadro e atingir a meta de redução da não adesão dos docentes da FGV de $31 \%$ para $11 \%$.

A primeira ação a ser tomada será a criação de vídeos pílulas que serão enviados por email para os docentes, além de serem disponibilizados na página de login do ECLASS, mensalmente.Os vídeos pílulas terão como conteúdo apresentar casos de dificuldades diárias dos professores e como o ECLASS pode colaborar para solucionar ou melhorar a situação, como por exemplo, o quarto professor da pesquisa, relata a dificuldade de organizar todos os trabalhos recebidos impressos, a falta de verificação de plágio e como se pode economizar no tempo de correção dos trabalhos.

Outra ação a ser realizada será a realização de eventos com os professores da Fundação. Serão identificados professores chaves que fazem uso continuo e eficiente da plataforma e que poderão compartilhar suas experiências com os demais colegas. Esses professores serão multiplicadores do conhecimento. Os professores multiplicadores mostrarão para seus colegas os cases de sucesso que tiveram ao utilizar - ECLASS como apoio de suas aulas presenciais. Neste evento, também serão divulgados os novos recursos e seus possíveis usos, será feito um overview dos recursos existentes, a divulgação da área CTE e o apoio disponível para o uso do ECLASS.

A oferta de capacitações no uso dos recursos do ECLASS é outra ação que será realizada, mensalmente. Os professores serão encorajados a entrar em contato com a equipe responsável pelo ECLASS para agendar uma capacitação. Além disso, será realizado um mapeamento de como ocorre a contratação dos docentes nas oito escolas da Fundação Getulio Vargas.Com o mapeamento realizado será possível abordar os 
docentes logo no início de suas atividades, assim podendo oferecer e apresentar todo potencial do AVA, como também presenteá-los com um kit de "seja bem-vindo" onde se reforça os benefícios no uso do ECLASS como extensão da sala de aula.

\section{Conclusão}

A partir das respostas de 148 docentes da Fundação Getulio Vargas ao questionário que realizado com o intuito de encontrar explicações satisfatórias às perguntas elencadas no objetivo deste trabalho, foi concluído que os docentes que pouco utilizam o sistema ECLASS representam uma minoria dentro do total de respondentes à pesquisa. Apesar disso, foi possível identificar um motivo principal, dentre as variáveis estudadas pelo presente trabalho, como fator motivador à não adesão pela pequena parcela dos professores à nova plataforma. $O$ cruzamento das respostas referentes à frequência de uso do ECLASS com as demais informações fornecidas pelos professores, seguido da análise estatística de como as variáveis se relacionavam entre si, permitiram a rejeição das hipóteses comuns para explicar a não adesão à ferramenta (tais como idade e tempo de casa) e foi identificado a baixa percepção dos benefícios do ECLASS por alguns professores como principal determinante à pouca utilização da plataforma digital.

Foi possível distinguir que os docentes que pouco utilizam o ECLASS tendem a não perceber os benefícios que a plataforma proporciona no apoio às aulas presenciais. Houve a observação que a falta de percepção está diretamente relacionada à quantidade de funcionalidades do sistema ECLASS que os professores têm ciência. Portanto, o que a amostra trabalhada permite afirmar é que quanto menor 0 conhecimento dos professores em relação às funcionalidades do sistema ECLASS, menor tende a ser a percepção dos professores quanto aos benefícios que a plataforma pode oferecer no apoio às aulas presenciais e, assim, menor tende a ser a frequência de utilização do ECLASS pelo corpo docente.

As afirmações acima puderam ser verificadas quando foi aplicado a pesquisa exploratória com a amostragem de quatro específicos docentes que compunham o universo de professores que não fizeram a adesão ao ECLASS. Como evidenciado na pesquisa os docentes em sua maioria não faziam uso do ECLASS por desconhecer seus benefícios. Após essas constatações, foram feitas sugestões de ações mais eficientes para que se possa reduzir a não adesão dos docentes da FGV de 31\% para $11 \%$ em um ano e que apresentem de forma clara os benefícios com o uso do ECLASS no apoio a aula presencial.

"Este artigo expressa a opinião do autor, não representando necessariamente a opinião institucional da FGV" 